\title{
Evaluation of Isothermal Recombinase Polymerase Amplification Incubation Temperature Tolerance
}

\section{Overview}

Isothermal DNA/RNA amplification technology provides flexibility of use, particularly in the field. TwistDx's proprietary Recombinase Polymerase Amplification (RPA) biochemistry enables such field use. Firstly, it permits transport of reagents (freeze-dried enzymatic pellets) at room temperature to point of testing, even in remote areas. Secondly, it allows amplification and detection of DNA/RNA using low cost, portable equipment. RPA works at an optimal temperature range of $39-42^{\circ} \mathrm{C}$, typically in 3-15 minutes. Low temperature amplification reduces hardware requirements. The tolerance to further reduced temperatures was explored.

\section{Materials and Methods}

TwistAmp ${ }^{\oplus}$ Basic kit (50 $\mathrm{\mu l}$ ) reactions were prepared as follows:

- Each freeze-dried pellet was rehydrated with $29.5 \mu$ rehydra-

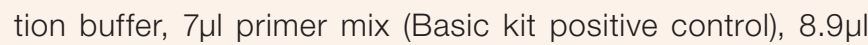
$\mathrm{dH} 20,3.6 \mu \mathrm{l}$ magnesium acetate $(280 \mathrm{mM})$, $1 \mu \mathrm{l}$ of positive control template (250 copies/ul) or $1 \mu \mathrm{l} \mathrm{dH20} \mathrm{for} \mathrm{no} \mathrm{template}$ control (ntc).

- Reactions were incubated for an hour at a temperature of 25 , $30,35,40$, or $45^{\circ} \mathrm{C}$ in a heat block, reactions were briefly agitated after 4 minutes incubation.

- Post amplification, reactions were cleaned with a standard PCR clean-up kit and run on a $2 \%$ agarose gel.

\section{Summary}

All reactions amplified a correctly sized product, at all the incubation temperatures tested. This flexible working temperature provides further evidence of RPA suitability for field work. A lower temperature requirement means lower power requirements key for field applications. Furthermore RPA technology can be applied simply in a lateral flow format (TwistAmp ${ }^{\circledR}$ nfo).

TwistAmp ${ }^{\circledR}$ users have previously reported similar flexibility in RPA temperature requirements with HIV $(1,2)$ and Schistosoma japonicum (3) targets.

The whole range of TwistAmp ${ }^{\circledR}$ products can be purchased at www.twistdx.co.uk/products or over the phone at $+44(0) 1223$ 496700 .

\section{References}

1. http://dx.doi.org/10.1371/journal.pone.0108189

2. http://dx.doi.org/10.1371/journal.pone.0112146

3. $h t t p: / / d x . d o i . o r g / 10.1186 / s 13071-016-1745-5$ 